\title{
A rare SMAD9 mutation identifies the BMP signalling pathway as a potential osteoanabolic target
}

Celia L Gregson* (PhD, FRCP), Musculoskeletal Research Unit, Translational Health Sciences, Bristol Medical School, University of Bristol, Bristol, UK

Dylan Bergen (PhD)†, (1) Musculoskeletal Research Unit, Translational Health Sciences, Bristol Medical School, University of Bristol, Bristol, UK. (2) School of Physiology, Pharmacology, and Neuroscience, Faculty of Life Sciences, University of Bristol, UK

Paul Leo (PhD)†, Translational Genomics Group, Institute of Health and Biomedical Innovation, Faculty of Health, Queensland University of Technology, Translational Research Institute, Princess Alexandra Hospital, Ipswich Rd, Woolloongabba, QLD 4102, Australia.

Richard B. Sessions (PhD), School of Biochemistry, Faculty of Life Sciences, University of Bristol, UK Lawrie Wheeler (BSc) Translational Genomics Group, Institute of Health and Biomedical Innovation, School of Biomedical Sciences, Queensland University of Technology (QUT), Translational Research Institute, 37 Kent St, Woolloongabba, QLD, 4102.

April Hartley (BSc) (1) Musculoskeletal Research Unit, Translational Health Sciences, Bristol Medical School, University of Bristol, Bristol, UK, (2) Medical Research Council Integrative Epidemiology Unit, Population Health Sciences, Bristol Medical School, University of Bristol, UK

Scott Youlten (PhD) Division of Bone Biology, Garvan Institute of Medical Research, Sydney, NSW 2010, Australia

Peter I Croucher (PhD) (1) Division of Bone Biology, Garvan Institute of Medical Research, Sydney, NSW 2010, Australia, (2) St Vincent's Clinical School, Faculty of Medicine, UNSW Sydney, Sydney NSW 2010, Australia and (3) School of Biotechnology and Biomolecular Sciences, UNSW Sydney, Sydney, NSW 2052, Australia

Aideen M. McInerney-Leo (PhD) (1) Dermatology Research Centre, The University of Queensland, The University of Queensland Diamantina Institute, Brisbane, Queensland, Australia. (2) Translational Genomics Group, Institute of Health and Biomedical Innovation, School of Biomedical Sciences, Queensland University of Technology (QUT), Translational Research Institute, 37 Kent St, Woolloongabba, QLD, 4102, Australia

William Fraser (MD) (1) Norwich Medical School, University of East Anglia, Norwich Research Park, Norwich, UK. (2) Departments of Diabetes, Endocrinology and Clinical Biochemistry, Norfolk and Norwich University Hospital NHS Foundation Trust, Colney Lane, Norwich, UK

Jonathan C.Y. Tang (MSc) Norwich Medical School, University of East Anglia, Norwich Research Park, Norwich, UK

Lisa Anderson (B.BiomedSci) Translational Genomics Group, Institute of Health and Biomedical Innovation, School of Biomedical Sciences, Queensland University of Technology (QUT), Translational Research Institute, 37 Kent St, Woolloongabba, QLD, 4102, Australia

Mhairi Marshall (MSc) Translational Genomics Group, Institute of Health and Biomedical Innovation, School of Biomedical Sciences, Queensland University of Technology (QUT), Translational Research Institute, 37 Kent St, Woolloongabba, QLD, 4102, Australia

Leon Sergot (FRCR) Severn School of Radiology, Severn Deanery, Park House, Bristol, UK 
Lavinia Paternoster (PhD), Medical Research Council Integrative Epidemiology Unit, Population Health Sciences, Bristol Medical School, University of Bristol, UK

George Davey-Smith (MD) Medical Research Council Integrative Epidemiology Unit, Population Health Sciences, Bristol Medical School, University of Bristol, UK

The AOGC Consortium

Matthew A Brown (MD, FRACP) (1) Translational Genomics Group, Institute of Health and Biomedical Innovation, Faculty of Health, Queensland University of Technology, Translational Research Institute, Princess Alexandra Hospital, Ipswich Rd, Woolloongabba, QLD 4102, Australia Chrissy Hammond (PhD), School of Physiology, Pharmacology, and Neuroscience, Faculty of Life Sciences, University of Bristol, UK

John P Kemp (PhD) (1) The University of Queensland Diamantina Institute, Translational Research Institute, University of Queensland, Brisbane, QLD 4102, Australia, (2) Medical Research Council Integrative Epidemiology Unit, Population Health Sciences, Bristol Medical School, University of Bristol, UK

Jon H Tobias+ (FRCP), Musculoskeletal Research Unit, Translational Health Sciences, Bristol Medical School, University of Bristol, Bristol, UK

Emma L Duncan+ (PhD) (1) Department of Endocrinology and Diabetes, Royal Brisbane \& Women's Hospital, Butterfield St, Herston, QLD 4029, Australia. (2) Translational Genomics Group, Institute of Health and Biomedical Innovation, Faculty of Health, Queensland University of Technology, Translational Research Institute, Princess Alexandra Hospital, Ipswich Rd, Woolloongabba, QLD 4102, Australia. (3) Faculty of Medicine, University of Queensland, Herston, QLD 4006, Australia.

† Joint second authors

+ Joint last authors

*Corresponding author: Dr Celia L Gregson, Musculoskeletal Research Unit, Translational Health Sciences, Bristol Medical School, University of Bristol, Learning \& Research Building (Level 1), Southmead Hospital, Bristol, BS10 5NB, UK. Tel: 00441174147842.

Email: celia.gregson@bristol.ac.uk

\section{Word counts}

Abstract: 150

Main text: 3752

Methods: 2380 


\begin{abstract}
To identify targets for novel anabolic medicines for osteoporosis, we recruited a large cohort with unexplained high bone mass (HBM). Exome sequencing identified a rare (minor allele frequency 0.0014) missense mutation in SMAD9 (c.65T>C, p.Leu22Pro) segregating with HBM in an autosomal dominant family. The same mutation was identified in another two unrelated individuals with HBM. In-silico protein modelling predicts the mutation severely disrupts the MH1 DNA-binding domain of SMAD9. Affected individuals have bone mineral density [BMD] Z-Scores +3 to +5 , with increased volumetric cortical and trabecular BMD, increased cortical thickness, and low/normal bone turnover. Fractures and nerve compressions are not seen. Both genome-wide, and gene-based association testing of heel estimated-BMD in $>362,924$ UK-Biobank British subjects showed strong associations with SMAD9 ( $\left.\mathrm{P}_{\mathrm{GWAS}}=6 \times 10^{-16} ; \mathrm{P}_{\mathrm{GENE}}=8 \times 10^{-17}\right)$. Smad9 is highly expressed in murine osteocytes and zebrafish bone tissue. Our findings support SMAD9 as a novel HBM gene, and a potential novel osteoanabolic target.
\end{abstract}

Keywords: High Bone Mass, SMAD9, DXA, exon sequencing, monogenic, zebrafish 


\section{Background}

Age-related bone loss with deterioration of skeletal architecture leads to osteoporosis, affecting 8.2 million women and 2.0 million men aged 50 years and older in the United States (US) ${ }^{1}$. Worldwide, osteoporosis causes more than 8.9 million fractures annually ${ }^{1}$. Osteoporotic fractures and their treatment are a major cause of morbidity and mortality, with annual US healthcare costs exceeding $\$ 20$ billion ${ }^{2}$. Most osteoporosis treatment approaches, including all oral medications, reduce bone resorption and prevent further bone loss, rather than enhance bone formation. Affordable anabolic treatments, which can restore bone mass and skeletal architecture, are much needed.

Romosozumab, a monoclonal antibody against sclerostin, represents a new class of anti-osteoporosis drugs ${ }^{3,4}$ (licensing currently under review). Sclerostin, a key inhibitor of bone formation, was discovered through study of two rare syndromes of extreme high bone mass (HBM) due to mutations in SOST ${ }^{5,6}$. SOST encodes Sclerostin which binds to low-density lipoprotein receptor-related proteins 5 and 6 (LRP5 and LRP6) to prevent activation of canonical WNT signalling in bone, resulting in decreased bone formation. Gain-of-function mutations in $L R P 5$ and $L R P 6$ can also cause extreme HBM 7,8 . Together these sclerosing bone dysplasias are characterised by mandible enlargement with tori of the palate and mandible, bone overgrowth leading to nerve compression, a tendency to sink when swimming, and, importantly, resistance to fracture ${ }^{5,7,9}$. These important gene discoveries validate the study of rare monogenic HBM as an approach to identify novel therapeutic targets for drug development towards osteoporosis treatments.

We have previously shown that HBM (defined as a total hip and/or $1^{\text {st }}$ lumbar vertebral bone mineral density [BMD] Z-score of $\geq+3.2$ ) is observed in $0.18 \%$ of Dual-Energy X-ray Absorptiometry (DXA) scans in the UK ${ }^{10}$. Most cases are unexplained, i.e. they do not carry mutations in established HBM genes ${ }^{9}$. Whilst such HBM populations do show enrichment for common variant associations with established BMD-associated loci ${ }^{11}$, we hypothesized that novel causes of monogenic HBM remain to be determined. Thus, we aimed to identify novel monogenic causes of HBM to provide insight into regulatory pathways amenable to therapeutic intervention. 


\section{Results}

\section{HBM pedigree with a segregating SMAD9 p.Leu22Pro mutation (Figure 1 and Table 1)}

We investigated a pedigree with unexplained and apparently autosomal dominant HBM $^{9}$, identified from our large UK HBM cohort ${ }^{10}$ (Figure 1 ).

\section{Clinical phenotype (Table 1)}

Clinical phenotypes of the UK family individuals are shown in Table 1; extended clinical histories are provided in Supplementary Results 1. In summary, affected individuals had high BMD Z-scores, and very high body mass index (BMI); and had not have any adult fractures, nerve compression or dental problems; however, bone pain was common, without a clear cause. There was no clinical history of intellectual impairment, pulmonary hypertension, vascular hypertension, haematological abnormalities, pubertal delay or other clinical conditions. None had been exposed to anabolic or antiresorptive medications.

\section{III.1: Index Case (c.65T>C, p.Leu22Pro)}

The 33-year-old index case, with BMD Z-Scores +3.2 at the total hip and +4.5 at L1, had only sustained one traumatic fracture aged 20 months. She reported lower leg and ankle pain. She was tall ( $>97^{\text {th }}$ centile) and obese, with increased shoe size, a broad frame, enlarged mandible and a $4 \mathrm{~mm}$ torus mandibularis. She had normal joints. Radiographs showed increased cortical thickness and new bone formation at the anterior inferior iliac spines bilaterally (Supplementary Figure 1).

\section{II.2: Mother of the index case (c.65T>C, p.Leu22Pro)}

The 55-year-old affected mother, with BMD Z-Scores +3.3 at the total hip and at L1, had never sustained a fracture. Six years earlier she had had a right calcaneal spur surgically removed. She had widespread pain with a diagnosis of fibromyalgia. She was tall ( $97^{\text {th }}$ centile) and obese, with above average shoe size, a broad frame, enlarged mandible but no tori. She had a full range of movement in all joints, bilateral knee crepitus and bilateral pes planus.

\section{III.2: Half-sister to index case (c.65T>C, p.Leu22Pro)}

The 22-year-old affected half-sister, with BMD Z-Scores +4.8 at the total hip and +2.6 at L1, had not fractured. She had had sciatica for five years, lumbar back pain and fronto-temporal headaches for 11 years, with a diagnosis of migraine. She was tall $\left(93^{\text {rd }}\right.$ centile) and obese, with above average shoe size, a broad frame, enlarged mandible, a torus palitinus in the midline of her hard palate $(3 \mathrm{~cm} x$ $7 \mathrm{~mm}$ ) and normal joint movement.

\section{2: Grandmother of index case (wild-type)}

The 75-years-old grandmother, who did not have HBM, had also never sustained a fracture. She had widespread osteoarthritis and on examination had reduced extension of the right elbow and left knee, and bilateral knee crepitus. However, in contrast to other family members, she was overweight with normal shoe size, a normal frame, mandible and no tori.

\section{Sequencing of pedigree}

Whole exome sequencing (WES) identified a heterozygous missense variant in SMAD9 (SMAD family Member 9 referring to homologies to the Caenorhabditis elegans SMA (small worm phenotype) and Drosophila MAD ("Mothers Against Decapentaplegic")) (NM 001127217: exon2: c.65T>C, p.Leu22Pro), segregating with HBM (i.e. present in all three individuals with HBM (III.1, II.2, III.2), but absent from I.2 (Figure 1). This variant (rs111748421) is rare (Exome Aggregation Consortium [ExAC] minor allele frequency [MAF] 0.0014), affects a highly evolutionarily conserved base (genomic evolutionary rate profiling [GERP] 5.53) and is predicted to be pathogenic by multiple protein- 
prediction algorithms (deleterious by SIFT ${ }^{12}$, probably damaging by Polyphen ${ }^{13}$, and disease causing by MutationTaster ${ }^{14}$ and PMut ${ }^{15}$ ).

A novel variant in CHRNA1 (cholinergic receptor, nicotinic, alpha 1) (c.560T>C, p.Leu187Pro) was also identified ( GERP 5.29). Mutations in CHRNA1 have been associated with congenital myasthenic syndromes (OMIM\#100690), not present in this pedigree.

No variants were identified when applying a compound heterozygous or an autosomal recessive inheritance model.

\section{Sequencing of other HBM cases identifies two further isolated HBM cases harbouring a p.Leu22Pro mutation \\ WES of a further $366 \mathrm{HBM}$ cases ( 240 isolated cases from the UK cohort with a total hip (TH) or first lumbar vertebra (L1) Z-score $\geq+3.2$ and 126 individuals from the Anglo-Australasian Osteoporosis Genetics Consortium (AOGC) ${ }^{16}$ with either a total hip and/or lumbar spine (LS) Z-score between +2.5 and +4.0) (Supplementary Figure 2) identified two individuals with the same SMAD9 c.65T>C, p.Leu22Pro mutation. Haplotypic analysis confirmed these women were neither related to each other nor to the pedigree described above.}

\section{Clinical phenotype \\ Isolated HBM case (c.65T>C, p.Leu22Pro) from the UK}

(Table 1; Supplementary Figure 3; Supplementary Results 1). This 55-year-old female, with BMD ZScores +5.0 at the total hip and +4.7 at $L 1$, had never fractured and had no symptoms of nerve compression. Her adult left upper cuspid tooth had never erupted; wisdom teeth had been extracted for overcrowding. She had noticed her own mandible enlargement. She had a congenital astigmatism of her left eye with poor vision, and congenital bilateral pes planus. Height was normal $\left(30^{\text {th }}\right.$ centile). She was obese with a broad frame, mandible enlargement, but no tori. She had normal joints.

\section{Isolated HBM case (c.65T>C, p.Leu22Pro) from Australia}

This 57-year-old female, with BMD Z-Scores +3 . at the total hip and +2.7 at L1, reported a nose fracture as a child. Height was on the $45^{\text {th }}$ centile and she was overweight. She did not have any history of conditions affecting bone health; and had not received antiresorptive or anabolic medications. No further clinical details were available.

\section{Tibial pQCT evaluation}

All members of the HBM pedigree, plus the additional isolated HBM case from the UK underwent pQCT scanning of the tibia (Table 2, Supplementary Table 1, Figure 2). To set these findings in context, the mean (SD) values from the four p.Leu22Pro SMAD9 HBM cases were compared against values from 76 unrelated female HBM cases (without SMAD9, LRP5, LRP4, or SOST mutations) and 32 female family controls with normal DXA-measured BMD who had had PQCT scans following the same protocol ${ }^{17}$. In addition to greater bone size (cross-sectional bone area) at the mid tibia $(66 \%$ site), the four HBM cases with SMAD9 mutations had greater trabecular and cortical density, cortical area and thickness and predicted bone strength (strength stain index [SSI]) than other HBM cases, and, to a greater extent, than unaffected family controls (with normal DXA-measured BMD (Table 2)). Muscle size (cross-sectional area) was also notably larger in in the SMAD9 HBM group (Figure 2).

\section{Sequencing of Low Bone Mass (LBM) cases}

WES data from 473 women with LBM from the AOGC consortium with TH Z-scores between -1.5 and -4.0 and a LS Z-score $\leq-0.5$, obtained using similar methodology to the AOGC HBM cases, was interrogated (Supplementary Figure 2). The p.Leu22Pro SMAD9 mutation was not observed. 


\section{Common SMAD9-associated genetic variants and BMD}

Publicly available data from a recent population-based genome-wide association study (GWAS) of eBMD (estimated BMD by heel ultrasound in the UK-Biobank study) ${ }^{18}$ were used to investigate variants surrounding both SMAD9 and CHRNA1. Regional association plots suggested that SNPS intersecting SMAD9 are strongly associated with eBMD (lead SNP rs12427846 [MAF 0.25], $\beta$ 0.02, SE $0.002, \mathrm{p}=5.5 \times 10^{-16}$; Figure 3$)$. In contrast, SNPs surrounding CHRNA1 were not robustly associated with eBMD (Supplementary Figure 4). These observations were further supported by gene-based tests of association performed in-house using 362,924 unrelated white British subjects from the UKBiobank Study. Specifically, SMAD9 was more strongly associated with eBMD ( $\left(\mathrm{P}_{\mathrm{JoNNT}}=7.94 \times 10^{-17}\right)$, when compared to neighbouring genes within $+/-800 \mathrm{~kb}\left(\mathrm{P}>2.4 \times 10^{-2}\right)$ (Supplementary Table 2). No such enrichment was seen for CHRNA1 (Supplementary Table 3). Further investigation of rs12427846 in the UK-Biobank study identified weak associations with body weight $(\beta-0.14$, SE 0.04 , $\left.p=1.6 \times 10^{-3}\right)$ and with height $\left(\beta-0.07\right.$, SE $\left.0.03, p=3.5 \times 10^{-3}\right)$ with effects in the opposite direction from that seen with eBMD; however, adjustment for weight and height did not attenuate the strong association between rs12427846 and eBMD reported above. Using PhenoScanner version $2{ }^{19}$, a PheWAS for the rs12427846 variant further identified a weak association with forearm BMD with consistent direction of effect (Supplementary Table 4). Interrogating the GWAS Catalog (https://www.ebi.ac.uk/gwas/), no associations with any trait (neither bone-related nor any other) have been reported previously for the rare (MAF=0.0014) variant $r s 111748421$.

\section{Smad9 expression in murine osteocytes}

We next determined whether Smad9 and Chrna1 are expressed in osteocytes, the master cell regulators in the skeleton and key regulators of bone mass ${ }^{20}$, and enriched in osteocytes compared to other cells in bone ${ }^{21}$. Smad9 mRNA was highly expressed in murine osteocytes whilst Chrna1 was not (Supplementary Table 5).

\section{Smad9 expression in zebrafish skeletal tissue}

We also examined Smad9 protein expression in the developing zebrafish skeleton ${ }^{22}$ at 6 - and 7-days post fertilisation (dpf) (Figure 4A). A focus of Smad9 expression was observed at the dorsal tip of the opercle, an intramembranous bone overlying the gills, adjacent to but distinct from a region of bone morphogenetic protein (BMP) reporter activity (Figure 4B). The opercula muscle group also showed evidence of BMP reporter activity, whereas Smad9 expression at this site was absent. Smad9expressing cells in the opercle were negative for the osteoblast marker, sp7 (osterix), suggesting they are likely to represent pre-osteoblasts (Figure $4 \mathrm{C}$ and Supplementary video 1 ). Equivalent findings were observed in the branchiostegal ray bones and in the notochord at 6- and 7- dpf (Supplementary Figure 5).

\section{SMAD9 protein structural modelling}

SMAD9 is a TGF-beta family member DNA binding transcription factor. Phosphorylation by BMPligand-bound type 1 receptor kinase activates SMAD9, which translocates from the cytoplasm to the nucleus to regulate target gene expression ${ }^{23}$. The seven exons of human SMAD9 encode a protein of 467 amino acids that contains two MAD-homology (MH) domains (MAD: Mother against Dpp) separated by a linker region (Figure 5). The p.Leu22Pro SMAD9 mutation is located within the MH1 domain responsible for DNA-binding (Figure 5 ), and lies in the hydrophobic face of the N-terminal alpha helix (helix-1) (Figure 6, Supplementary Video 2). Helix-1 packs against a groove made by helix2 and -3 within $\mathrm{MH1}$, forming part of the hydrophobic core of this domain. Substitution of leucine by proline will: a) introduce a less hydrophobic residue into this position; and b) compromise the $\alpha$ helical fold by disrupting the canonical hydrogen bonding of helix-1. Thus, modelling suggests that this mutation will disrupt the MH1 domain so severely that SMAD9 can no longer bind DNA and/or will be unstable leading to protein degradation. 


\section{Discussion}

We report the first HBM pedigree with a segregating SMAD9 mutation, with replication in two further unrelated individuals with HBM. SMAD9 (also known SMAD8, MADH6, and MADH9) encodes a downstream modulator of the BMP signalling pathway. BMPs, members of the TGF- $\beta$ superfamily, were first reported in 1977 to induce the formation of bone and cartilage when transplanted subcutaneously ${ }^{24}$. SMADs, activated by ligand-binding of cell surface BMP receptors, mediate downstream intracellular signalling and biological responses induced by BMPs ${ }^{25}$. Smad6 and Smad7 both inhibit BMP receptor activation and downstream signalling, as does Smad9 by more direct transcriptional repression ${ }^{23}$. Our in-silico protein modelling predicts that the p.Leu22Pro mutation severely disrupts the structure of the MH1 DNA binding domain of SMAD9, leading to loss of function.

Whilst few previous studies have examined sites of Smad9 tissue expression, we have confirmed that Smad9 is expressed in both mouse cortical bone derived osteocytes, and in skeletal elements of zebrafish larvae. Moreover, we observed that BMP reporter activity in zebrafish was absent at sites of Smad9 expression, consistent with a functional role in BMP repression ${ }^{23}$. Taken together our findings suggest that SMAD9 c.65T>C is a loss-of-function mutation, causing $\mathrm{HBM}$ through a novel mechanism of enhanced bone formation as a consequence of reduced BMP inhibition.

Further, we have shown that the region containing SMAD9 is strongly associated with BMD within the general population. Common variants intersecting SMAD9 associate with population-based measures of eBMD, as evidenced recently ${ }^{18,26}$ and from our gene-based tests of association presented here. Furthermore, rs 12427846 [the lead SNP from these eBMD results] is associated with DXA-measured total body BMD ${ }^{27}$ and fracture risk ${ }^{18}$. These findings, which provide further evidence of the importance of SMAD9 in bone biology, are equivalent to reported associations for common variants annotated to $L R P 5$ and SOST genes, both similarly implicated in monogenic HBM disorders ${ }^{28,29}$.

We have previously estimated unexplained HBM to have a prevalence of $0.181 \%$ amongst a DXAscanned adult population in the UK ${ }^{10}$. As two of 248 cases fulfilling our stringent HBM phenotype definition (Supplementary Figure 2) were found to harbour the p.Leu22Pro mutation, we would estimate SMAD9 HBM to have a prevalence of approximately 1 in 100,000 $\left(1.46 \times 10^{-5}\right)$; less common than $\angle R P 5 \mathrm{HBM}^{9}$.

The clinical phenotype of p.Leu22Pro SMAD9 HBM includes mandible enlargement, a broad frame, torus palitinus, pes planus, increased shoe size and a tendency to sink when swimming. Adult fractures were not reported, raising the possibility of increased skeletal strength which would be supported by greater cortical bone and an increased strength-strain index (SSI) quantified by pQCT (discussed further below), both of which promote fracture resistance. Mandible enlargement, torus palitinus, a tendency to sink when swimming, and an absence of adult fractures are reminiscent of $L R P 5 \mathrm{HBM}^{7,30}$. Encouragingly, unlike sclerosteosis (due to anabolic SOST mutations) and some LRP5 HBM cases ${ }^{9,31}$, nerve compression does not seem to be a feature of SMAD9 HBM.

The musculoskeletal phenotype of p.Leu22Pro SMAD9 HBM includes high BMD Z-Scores $(+3$ to +5$)$, with increased fat and lean mass. PQCT revealed that both volumetric cortical and trabecular bone density are increased. Furthermore, the larger bone size with greater cortical thickness and cortical area, suggest a possible combination of enhanced modelling to increase periosteal expansion and reduced bone remodelling to reduce endosteal expansion. In support, bone turnover markers are at the lower end of the normal range. This phenotype also mimics that previously described for human $L R P 5 \mathrm{HBM}^{32}$. Plasma sclerostin is not elevated, in contrast to $L R P 5 \mathrm{HBM}^{33}$, suggesting that a negative feedback loop downregulating WNT signalling is not present. 
PQCT further confirmed that larger muscle size is another phenotypic feature of SMAD9 HBM, with an increase in muscle cross-sectional area. This contrasts with the suggestion from our zebrafish studies that Smad9 expression in muscle tissue may be limited. Given the well-recognised cross-talk between muscle and bone ${ }^{34}$ and the large BMI of these individuals, it is conceivable that the increase in muscle size is a consequence of increased bone mass. However, similar increases in muscle size have not been reported in other monogenic HBM conditions (i.e. LRP5 or SOST HBM) with equivalent increases in BMD.

Heterozygous truncating SMAD9 mutations are associated with primary pulmonary hypertension (OMIM\#615342) ${ }^{35}$, a phenotype not apparent in our HBM cases. Reported mutations affect a different domain from the mutation observed here (p.Leu22Pro), with p.Cys202X ${ }^{35}$ and p.Arg294X ${ }^{36}$ truncating the SMAD9 protein in the linker region between $\mathrm{MH} 1$ and $\mathrm{MH} 2$. A truncating mutation (p.Arg247X) has been associated with cerebral arteriovenous malformations in childhood ${ }^{37}$. An activating heterozygous p.Val90Met germline mutation, affecting the $4^{\text {th }} \alpha$-helix of $\mathrm{MH} 1$ and close to the DNA binding interface, has been described in one pedigree with hamartomatous polyposis ${ }^{38}$. In contrast to p.Leu22Pro, p.Val90Met appears to be a gain-of-function mutation, thought to arise from a steric clash, prompting a His104 residue to enhance DNA binding ${ }^{38}$. Such examples of diverse phenotypes arising from mutations in differing exons of the same gene are well recognised, e.g. differing mutations in FBN1 (Fibrillin 1) can cause Marfan syndrome (with associated tall stature) (OMIM\#154700), acromicric dysplasias (with short stature) (OMIM\#102370), or stiff skin syndrome (OMIM\#184900) ${ }^{39-41}$. Similarly mutations in PIGN (Phosphatidylinositol Glycan Anchor Biosynthesis Class $N$ ) are associated with multiple congenital anomalies-hypotonia-seizures syndrome 1 (OMIM\#614080) ${ }^{42}$ and Fryns syndrome (congenital diaphragmatic hernia and other dysmorphic features) (OMIM\#229850) ${ }^{43}$.

We are only aware of one other skeletal dysplasia reported in association with an inhibitory SMAD (which include SMAD6 and SMAD7). A rare SMAD6 mutation has been associated with susceptibility to non-syndromic midline craniosynostosis 7 (OMIM\#617439) - but only in the context of coinheritance of a common variant in BMP2 strongly associated with this condition, a rare example of two locus inheritance ${ }^{44}$. Interestingly, amongst the 1103 conditionally independent SNPs reaching genome-wide significance in the UK-Biobank eBMD GWAS (population $n=426,824$ ), as well as identifying the SMAD9 locus, four novel SNPs annotating to SMAD7 were also reported (in addition to three established SNPs associated with SMAD3), all suggesting variation in inhibitory SMADs is likely of functional importance in human biology ${ }^{18}$.

The phenotype we describe here contrasts with that of activating mutations of the BMP receptor, ACVR1, which increase BMP signalling. However, in contrast to p.Leu22Pro SMAD9 HBM, ACVR1 mutations lead to a fatal condition, Fibrous Ossificans Progressiva (FOP, OMIM\#135100) ${ }^{45}$. In FOP, muscle tissue differentiates into bone following trivial injury, resulting in the formation of mature bone at multiple extra-skeletal sites. ACVR1 mutations may produce a more severe phenotype, compared with loss-of-function mutations in SMAD9 reported here, since ACVR1 also activates nonSMAD dependent BMP signalling cascades such as the NF-KB and p38 MAP kinase (p38MAPK) pathways, which are upregulated in FOP ACVR1 R206H monocytes ${ }^{46}$.

In view of the benign phenotype which we observed in p.Leu22Pro SMAD9 carriers, our findings suggest that SMAD9 is worthy of consideration as a drug target for osteoporosis. Our zebrafish studies suggest that Smad9 is expressed in pre-osteoblasts, consistent with the profile of an anabolic target capable of stimulating new bone formation through recruitment of early osteoblast progenitors. Given the pathological consequence of excess BMP activation in FOP, this pathway has not previously been prioritised as a possible target in osteoporosis research, despite the profound 
bone anabolic potential. Interestingly, phosphorylation of Smad9, as part of the Smad1/5/9 heterotrimer, has been researched in relation to fracture healing and bone regeneration: G-proteincoupled receptor kinase 2-interacting protein-1 (GIT1), a shuttle protein in osteoblasts, has been shown to regulate Smad1/5/9 phosphorylation which then mediates BMP2 regulation of Runx2 expression and thus endochondral bone formation at the site of fracture ${ }^{47,48}$. Moreover, local BMP administration has been used to promote bone repair following surgery ${ }^{49}$. Based on our findings, it is tempting to speculate that treatments intended to suppress SMAD9 activity might prove useful in treating osteoporosis, fractures, and possibly also sarcopenia.

Our study has limitations. All individuals with p.Leu22Pro SMAD9 HBM were female, which reflects the study design which favoured those with a historical DXA scan who are more likely to be female. Whether findings will be similar in men is unknown, although no sex-gene interaction has been described for the LRP4, LRP5, LRP6, or SOST sclerosing bone dysplasias. In the recent UK-Biobank eBMD GWAS, LD score regression analyses suggested that the genetic architecture influencing male and female eBMD was largely shared but that there were some significant differences between the sexes $(\mathrm{rG}=0.91, \mathrm{SE}=0.012, \mathrm{p}<0.001)^{18}$, consistent with earlier epidemiological studies ${ }^{50}$. The small sample of SMAD9 HBM cases ( $n=4$ with $p Q C T$ ) limited our ability to robustly evaluate associations statistically. The p.Leu22Pro mutation is a reported SNP with an rsID, indicating this is carried within the general population (e.g. in the UK at estimated 92,428 people might be expected to carry this mutation). This may be the case, given there is no indication that the phenotype affects reproductive fitness, and none of the phenotypic characteristics are deleterious to health; and HBM will not be overt unless a DXA scan is performed. The absence of reported association for rs 111748421 in the GWAS catalogue is not surprising given the difficulty of imputing extremely rare variants/ mutations for analysis in GWAS. Our GWAS was based on estimated heel BMD quantified by ultrasound rather than DXA-measured BMD. Estimated heel BMD is not used routinely in clinical practice. However, we have previously demonstrated a strong overlap between genetic loci identified by eBMD GWAS and by DXA-measured BMD GWAS ${ }^{26}$.

\section{Conclusions}

We report SMAD9 as a novel HBM-causing gene. The clinical phenotype of p.Leu22Pro SMAD9 HBM has many features in common with that of $L R P 5$ HBM, but lacks the deleterious features which characterise SOST HBM (sclerosteosis). As has been reported for both LRP5 and SOST, we demonstrate that SMAD9 can be disrupted by a single point mutation causing an extreme bone phenotype and perturbed by common variation affecting bone density within the general population. The role of SMAD9 in bone biology is supported by our finding of high levels of Smad9 expression in murine osteocytes, and in skeletal elements of zebrafish larvae. Smad9 is thought to inhibit BMP signalling to reduce osteoblast activity; thus, we hypothesise SMAD9 c.65T >C is a loss-offunction mutation reducing BMP inhibition, ultimately leading to enhanced bone formation. Our findings support SMAD9, and its role within the SMAD9-dependent BMP signalling pathway, as a potential novel anabolic target for osteoporosis therapeutics which warrants further investigation. 


\section{Methods}

\section{The UK HBM Cohort}

The HBM study is a UK-based multi-centred observational study of adults with unexplained HBM, identified incidentally on routine clinical DXA scanning. Full details of DXA database screening and participant recruitment have previously been reported ${ }^{10}$. In brief, DXA databases containing 335,115 DXA scans were initially searched for a BMD T or Z-score $\geq+4$ at any site within the LS or hip, at 13 UK centers. All 1505 DXA images were visually inspected; 962 cases with established and/or artefactual causes of raised BMD were excluded (including exclusion of artefact from LS osteoarthritis (see Supplementary Methods 1). As a generalized HBM trait should affect both spine and hip BMD, though not necessarily equally, HBM was defined as a) L1 Z-score of $\geq+3.2$ plus TH Z-score of $\geq+1.2$ and/or b) TH Z-score $\geq+3.2$ plus L1 Z-score of $\geq+1.2$ (using age and gender-adjusted BMD Z-scores). A threshold of +3.2 was in keeping with the only published precedent for identifying HBM using DXA ${ }^{7}$. Z rather than T-score was used to limit age bias.

\section{Recruitment}

533 unexplained HBM index cases were invited to participate. $248(47 \%)$ were recruited ${ }^{10}$. Index cases were asked to pass on study invitations to their first-degree relatives and spouse/partner(s). These relatives and spouses were invited only once, and non-responders were not followed-up. Relatives/spouses with HBM were in turn asked to pass on study invitations to their (previously uninvited) first-degree relatives and spouses. 893 relatives were invited to participate, of whom 236 (26.4\%) were recruited. 217 spouses/ partners were invited to participate, of whom 61 (28.1\%) were recruited; 2 individuals invited 2 partners ${ }^{10}$. All participants were clinically assessed using a standardised structured history and examination data collection tool.

\section{DXA measurements}

Participants underwent DXA scanning (repeat in the case of index cases) using either GE Lunar Prodigy DXA (software version 13.2, GEHealthcare, Madison, WI, USA) or Hologic Discovery/W DXA (software version Apex 3.0, Hologic Inc. Bedford, MA, USA). Scans were acquired and analysed according to each manufacturer's standard scanning and positioning protocols as previously described ${ }^{51}$. Total Body (TB) bone mineral content (BMC) and density (BMD), Fat Mass (FM) and Lean Mass (LM) were measured, together with L1 and total hip BMD. All DXA images were reviewed for quality control purposes.

\section{Peripheral Quantitative Computed Tomography (pQCT)}

pQCT scans were performed at the distal and mid-shaft of the tibia $(4,14,38 \& 66 \%$ from the distal endplate) in the non-dominant lower limb using a Stratec XCT2000L (Stratec Medizintechnik, Pforzheim, Germany); voxel size $0.5 \mathrm{~mm}$, CT speed $30 \mathrm{~mm} /$ second, XCT software version $5.50 \mathrm{~d}$; as published previously ${ }^{17}$. In brief, a reference line at the distal endplate was determined from initial frontal scout view. Cortical bone was defined using a threshold above $650 \mathrm{mg} / \mathrm{cm}^{3}$ (optimal for bone geometry ${ }^{52}$ ). Trabecular bone was identified by elimination of cortical bone and therefore trabecular bone mineral density (tBMD) was defined as a density $<650 \mathrm{mg} / \mathrm{cm}^{3}$. Cortical thickness, periosteal circumference and endosteal circumference were derived using a circular ring model. Further cortical parameters were measured: cortical bone mineral density (CBMD), total bone area (TBA) (i.e. total bone cross-section, reflecting periosteal expansion), cortical bone area (CBA) (reflecting a combination of periosteal and endosteal expansion) and CBA/TBA (\%). Strength Strain Index (SSI) was calculated according to Stratec's user manual (SSI $=\mathrm{SM}^{*}\left(\mathrm{CBMD}\left[\mathrm{mg} / \mathrm{cm}^{3}\right] /\right.$ $1200\left[\mathrm{mg} / \mathrm{cm}^{3}\right]$ ), where $1200 \mathrm{mg} / \mathrm{cm}^{3}$ represents the normal physiological density of bone (stated by Stratec) and SM (Section Modulus)=CSMI/ periosteal radius, where CSMI (Cross-Sectional Moment of Inertia $\left.\left[\mathrm{cm}^{4}\right]\right)=\Pi$ (periosteal radius ${ }^{4}$-endosteal radius $\left.\left.{ }^{4}\right) / 4\right)^{53}$. Twenty population controls were scanned twice on the same day after repositioning; measurement precision (CV) was typically 1 to 3 
$\%$ [11]. Stratec pQCT machines were calibrated using a COMAC phantom; mean (SD) difference between scanners was $1.18(0.82) \%$.

\section{Blood testing including bone turnover markers and DNA extraction}

Two non-fasted EDTA samples were collected and serum separated and frozen within 4 hours to $80^{\circ} \mathrm{C}$. Bone formation (Procollagen type 1 amino-terminal propeptide [P1NP], total osteocalcin) and resorption ( $\beta$-C-telopeptides of type I collagen $[\beta C T X]$ ) markers were measured by Electrochemiluminescence immunoassays (ECLIA) performed on the COBAS e601 analyser (Roche Diagnostics, Burgess Hill, UK). Inter-assay coefficient of variation (CV) for P1NP was $<3.0 \%$ across the range between $5-1200 \mu \mathrm{g} / \mathrm{L}$; osteocalcin was $<5.0 \%$ across the range between $0.5-300 \mu \mathrm{g} / \mathrm{L} ; \beta C T X$ was $<3.0 \%$ across the range between $0.01-6 \mu \mathrm{g} / \mathrm{L}$. Sclerostin was measured using an enzyme-linked immunosorbent assay (ELISA) kit BI-20492 (Biomedica GmbH, Vienna, Austria) with assay CV of $<9.0 \%$ across the range $2.6-240 \mathrm{pmol} / \mathrm{L}$. DNA was extracted from peripheral venous blood using standard phenol/ chloroform extraction.

\section{Exclusion of known monogenic causes of HBM}

Sanger sequencing of all HBM index cases for exons 2, 3 and 4 of LRP5, SOST (including the van Buchem disease deletion) and LRP4 (exons 25 and 26) excluded seven with LRP5 mutations and one with a SOST mutation ${ }^{9}$, leaving 240 unexplained HBM individuals.

\section{Ethics approval}

Written informed consent was collected for all participants in line with the Declaration of Helsinki ${ }^{54}$. This study was approved by the Bath Multi-centre Research Ethics Committee (REC: 05/Q2001/78) and at each NHS Local REC.

\section{Anglo-Australasian Osteoporosis Genetics Consortium (AOGC) HBM and LBM cases}

The original AOGC extreme truncate population included 1128 Australian, 74 New Zealand and 753 British women, aged between 55-85 years, five or more years postmenopausal, with either HBM (age and gender-adjusted BMD Z-scores of +1.5 to $+4.0, n=1055$ ) or LBM (age- and gender-adjusted BMD Z-scores of -4.0 to $-1.5, n=900)^{55}$. LBM cases were excluded if they had secondary causes of osteoporosis (as previously described ${ }^{55}$ ). Unrelated samples of Caucasian ancestry with complete height and weight data and enough high-quality genomic DNA were available in 947 AOGC individuals (426 AOGC high and 521 AOGC low BMD), from which (due to computation capacity limiting sample size) the most extreme HBM cases were then selected, using a threshold TH or LS Zscore $\geq+2.5$, and the most extreme LBM cases using a LS Z-Score $\leq-0.5$, so $126 \mathrm{HBM}$ and 493 LBM samples were chosen to undergo WES.

\section{Ethics approval}

The AOGC study was approved by the Queensland Office of Human Research Ethics Committee (Ref:2008/018), the University of Queensland (Ref:200800376) and/or relevant research ethics authorities at each participating centre. Directly recruited participants gave written, informed consent. Some participants were recruited through genetic and/or clinical studies (all with appropriate ethical approval) but also provided written informed consent to contribute to collaborative genetic studies ${ }^{56-58}$. DNA was obtained from peripheral venous blood or from saliva through standard methods.

\section{Whole exome sequencing}

Sequencing libraries for 859 samples (240 UK HBM, 126 AOGC HBM, 493 AOGC LBM) were constructed in two batches, determined by sample availability, the first ( 85 HBM, 619 AOGC) using the Illumina TruSeqDNA sample preparation kit, combined in pools of six for target capture by the Illumina TruSeq Exome v2.0 Enrichment Kit (64Mb capture) and assessed pre and post-capture for 
quality and yield with the Agilent High Sensitivity DNA assay and KAPA Library Quantification Kit. For the second batch (155 HBM), exome capture was performed using the Nextera ${ }^{\circledR}$ Rapid Capture Exome Enrichment Kit (Cat No FC-140-1003) (62Mb capture) (Illumina, San Diego, California, USA) as per the Nextera ${ }^{\circledR}$ Rapid Capture Enrichment. In both cases massive parallel sequencing was performed with six samples per flow cell lane on the Illumina HiSeq2000 platform and version 2 SBS reagents to generate $100 \mathrm{bp}$ paired-end reads. Base calling, sequence alignment and variant calling were all performed as previously described ${ }^{59}$, in brief, after demultiplexing, the Illumina Data Analysis Pipeline software (CASAVA v.1.8.2) was used for initial base calling. Sequence data were aligned to the current build of the human genome (UCSC Genome Browser, hg19, released February 2009) via the Novoalign alignment tool (v.2.08.02 1) ${ }^{60}$; sequence alignment files were converted by SAMtools (v.0.1.14) ${ }^{61}$ and Picard tools (v.1.42). SNPs and indels were called with the Genome Analysis Toolkit (GATK v.5506) ${ }^{62,63}$ and annotated by ANNOVAR ${ }^{64}$. Further analysis of sequence data was performed with custom scripts employing R and Bioconductor. Good-quality SNPs, excluding all those with a genotype quality score, determined by the GATK algorithm, $<60$ (range 0 [poor] to 100 [excellent]), were retained. Platform related artefacts variants were identified as variants where the allele count was 6 SDs higher than that expected from the maximum population MAF under the binomial approximation. Remaining SNPs and indels were assessed according to prediction of potentially damaging consequence ("nonsynonymous SNV," "splicing," "frameshift substitution," "stopgain SNV," "stoploss SNV") by using both RefSeq and UCSC transcripts. Further filtering excluded SNPs with a minor allele frequency (MAF) $<0.05$ (observed in NCBI dbSNP (GRCh37/ Hg19), 1000 Genomes ${ }^{65}$, ExAC (http://exac.broadinstitute.org/), or internal databases from >3000 exomes), 1000 Genomes small indels (called with the DINDEL program ${ }^{66}$ ). Variants not present in any of these databases were considered novel. Platform related artefacts were identified as variants where the allele count was 6 standard deviation higher than that expected from the maximum population MAF under the binomial approximation. Genotype Quality scores for the two additional isolated HBM cases were 99 and read depths were 15,14 and 21,19.

\section{Filtering pipeline applied to unexplained HBM pedigrees}

After quality-filtering as described above, data were analyzed for carriage of at least one rare (either novel or maximum population based MAF $<0.005$ ) nonsynonymous SNV or indel in a highly conserved region (GERP score $<1.5$ ) of a gene, carried by the affected individuals and not carried by unaffected individuals (i.e. autosomal dominant carriage model). Data were then filtered based on functional prediction of SNVs using Polyphen ${ }^{13}$ to identify 'probably damaging' and SIFT ${ }^{12}$ 'deleterious' SNVs. Compound heterozygous and homozygous inheritance were also assessed.

\section{Sanger sequencing validation of pedigree based HBM mutation}

Polymerase chain reaction (PCR) amplification of identified exons was performed on 50ng genomic DNA in a reaction mix consisting; $10 \mathrm{X}$ Immolase reaction buffer, $10 \mathrm{mM}$ dNTPs, $50 \mathrm{mM} \mathrm{MgCl}, 5 \mu \mathrm{M}$ each primer, $0.5 \mathrm{U}$ Immolase polymerase Taq (Bioline Reagents Ltd, London), and water to final volume of $25 \mu \mathrm{l}$. PCR cycling conditions and primer sequences are shown in (Supplementary Methods 2). Samples were Sanger sequenced using standard techniques (BigDye v3.1 chemistry, Life Technologies Corporation, California), and capillary sequenced (3130 Genetic Analyzer, Life Technologies Corporation, California). Electropherograms were aligned and analysed using sequence analysis software Genalys (Version 2.0 ß, Masazumi Takahashi).

\section{Multi-marker Analysis of GenoMic Annotation (MAGMA) in UK Biobank}

Gene-based tests of association were performed on 362,924 unrelated white British subjects (54\% female, GCTA-GRM derived pairwise relatedness $<0.10$ ) from the UK Biobank study that had valid quantitative ultrasound derived heel eBMD and high quality genome-wide HRC and 1000G/UK10K imputed data from the January 2018 release [i.e. 20,490,436 genetic variants with an information quality score $>0.3, \mathrm{MAF}>0.05$, minor allele count $>5$, genotyping hard call rate $>0.95$, and weak 
evidence of deviation from Hardy-Weinberg equilibrium $\left.\left(p>1 \times 10^{-6}\right)\right]$. A detailed description of the methodology used to generate this resource is published elsewhere ${ }^{18}$. Gene-based tests of association were implemented in MAGMA v1.06 ${ }^{67}$ using a multi-model approach that combines the association results from three separate gene analysis models: principal components regression, SNP-wise Mean $\chi 2$ model [i.e. test statistic derived as the sum of - $\log (\mathrm{SNP} p$-value)] and SNP-wise Top $\chi 2$ model [(test statistic derived as the sum of -log(SNP p-value) for top SNPs)] to produce an aggregate $p$-value corresponding to the strength of evidence of association between each of the 19,361 protein coding genes (+/- 20kb) and BMD, adjusting for age, sex, genotyping array, assessment centre and ancestry informative principal components $1-20$. Summary results statistics for all genes within +/- 800kb of SMAD9 and CHNR1 were looked up. Regional association plots were generated using LocusZoom (v1.3) ${ }^{68}$ in conjunction with summary association results from Morris et al $2018^{18}$.

\section{Gene expression in murine osteocytes}

Whole transcriptome sequencing data from the primary osteocytes of four different bone types (tibia, femur, humerus and calvaria) from mice (marrow removed, $n=8$ per bone) were analysed. A threshold of expression was determined based on the distribution of normalised gene expression for each sample ${ }^{69}$. "Expressed" genes were above this threshold for all 8 of 8 replicates in any bone type. Osteocyte enriched expression of these genes in the skeleton was determined by comparing transcriptome-sequencing data from bone-samples with osteocytes isolated versus those samples with marrow left intact ( $n=5$ per group) ${ }^{21}$.

\section{Replication in high BMD populations}

WES data from AOGC were analysed to identify any individual who carried the same rare (MAF $<0.025$ ) mutation as identified from analysis of the HBM pedigree. Polyphen ${ }^{13}$ and SIFT ${ }^{12}$, PMut ${ }^{15}$ and MutationTaster ${ }^{14}$ were used for in silico functional prediction. When the same point mutation was identified in more than one individual, haplotypes were compared between index case samples genotyped using an Infinium OmniExpress-12v1.0 GWAS chip read using an Illumina iScan (San Diego, California, USA), with genotype clustering performed using Illumina BeadStudio software.

\section{Protein structural modelling}

The amino-acid sequence of human SMAD9 was passed to the HHPred server (https://toolkit.tuebingen.mpg.de/\#/tools/hhpred) ${ }^{70}$. This located the best template structures in the Protein Databank for the MH1 domain, 5X6G (mouse SMAD5; 92\% identity), and the MH2 domain, 3GMJ (Drosophila melanogaster MAD; 75\% identity). Modeller was used to build the domain models according to the HHPred alignments ${ }^{71}$. Chimera was used to introduce point mutations and remodel the domain swapping in the SMAD9-MH1 model ${ }^{72}$.

\section{Zebrafish studies}

BMPre:GFP (Tg(5XBMPRE-Xla.Id3:GFP)) ${ }^{73}$ and sp7:GFP (Tg(Ola.sp7:NLS-GFP)) ${ }^{74}$ transgenic fish (in London $A B$ background) were maintained in standard conditions ${ }^{75}$. Experiments were approved by the University of Bristol Animal Welfare and Ethical Review Body (AWERB) and performed in accordance with a UK Home Office project license. Developmentally staged larvae (following euthanisation in MS222) were fixed in $4 \%$ paraformaldehyde (1 hour), dehydrated to $100 \%$ methanol and stored at $-20^{\circ} \mathrm{C}$ prior to staining. Immunolabelling protocol was as previously described ${ }^{76}$. Primary antibodies: anti-Smad9 (rabbit polyclonal, Abcam, ab96698) used at a 1/100 dilution and anti-GFP (chicken polyclonal, Abcam, ab13970) used at a 1/200 dilution in blocking buffer ( $5 \%$ horse serum). Secondary antibodies were used (A21206 and A11041, Invitrogen) in a 1/400 dilution and samples were incubated with DAPI (Sigma-Aldrich, 1/1000 dilution) to visualise nuclei. Samples were mounted in $1 \%$ low melting point agarose and images were taken with a confocal laser scanning microscope (Leica, SP5II AOBS attached to a Leica DM 16000 inverted epifluorescence microscope) 
using a 40X PL APO CS (1.3 numerical aperture) lens. Images were processed and colour balanced in $\mathrm{Fiji}^{77}$.

Supplementary Information is available in the online version of this paper.

\section{Acknowledgements}

Full acknowledgements are listed in the Supplementary Information.

\section{Funding}

CLG was funded by the Wellcome Trust (080280/Z/06/Z), the EU 7th Framework Programme under grant agreement number 247642 (GEoCoDE), a British Geriatric Society travel grant, and Versus Arthritis (formerly Arthritis Research UK) (grant ref 20000). This study was supported by the NIHR CRN (portfolio number 5163). DB received travel grants from The Harold Hyam Wingate Foundation, the Disease Models and Mechanisms journal (DMMTF-180208), and an Elizabeth Blackwell Institute for Health Research (University of Bristol) discipline hopping fellowship via a Wellcome Trust Institutional Strategic Support Grant (204813/Z/16/Z). AH is funded by the Wellcome Trust (grant ref 20378/Z/16/Z). LP, AH and GDS work in a unit which receives UK Medical Research Council funding (MC_UU_12013/4). CH and DB were funded by Versus Arthritis $(21211,21937,19476)$. AML is supported by an NHMRC Early Career Fellowship. JPK is funded by a University of Queensland Development Fellowship (UQFEL1718945) his contribution is supported by a National Health and Medical Research Council (Australia) project grant (GNT1158758). MAB is supported by an NHMRC Senior Principal Research Fellowship. PIC is funded by a Wellcome Trust Strategic Award (grant ref 101123). The AOGC was funded by the National Health and Medical Research Council (Australia) (Project Grants 511132 and 1032571).

\section{Author Contributions}

Conception CLG, GDS, MAB, JHT, ELD

Design CLG, GDS, MAB, PL, JHT, ELD

Data acquisition CLG, DB, LW, PC, SY, WF, JCYT, CH, MAB, ELD

Analysis CLG, DB, RBS, LW, AH, SY, PC, AML, CH, JPK, PL, ELD

Interpretation CLG, DB, RBS, AH, SY, PC, AML, MAB, CH, JPK, PL, JHT, ELD

Manuscript draft CLG, DB, LW, AH, JPK, LP, CH, JPK, PL, JHT, ELD

Manuscript revision CLG, DB, RBS, AH, AML, JCYT, LP, MAB, CH, JPK, PL, JHT, ELD

Approve final manuscript - all authors.

All authors take responsibility for their contributions as outlined above.

\section{Completing Interests}

Authors have no competing interest to declare 


\section{References}

$1 \quad$ Wright, N. C. et al. The recent prevalence of osteoporosis and low bone mass in the United States based on bone mineral density at the femoral neck or lumbar spine. J Bone Miner Res. 29, 2520-2526 (2014).

2 Burge, R. et al. Incidence and economic burden of osteoporosis-related fractures in the United States, 2005-2025. J Bone Miner Res. 22, 465-475 (2007).

3 McClung, M. R. et al. Romosozumab in Postmenopausal Women with Low Bone Mineral Density. New Eng J Med. 370, 412-420 (2014).

4 McClung, M. R. et al. Effects of 24 Months of Treatment With Romosozumab Followed by 12 Months of Denosumab or Placebo in Postmenopausal Women With Low Bone Mineral Density: A Randomized, Double-Blind, Phase 2, Parallel Group Study. J Bone Miner Res. 33, 1397-1406 (2018).

5 Brunkow, M. E. et al. Bone Dysplasia Sclerosteosis Results from Loss of the SOST Gene Product, a Novel Cystine Knot-Containing Protein. Am J Med Genet. 68, 577-589, (2001).

6 Staehling-Hampton, K. et al. A 52-kb deletion in the SOST-MEOX1 intergenic region on 17q12-q21 is associated with van Buchem disease in the Dutch population. Am J Med Genet 110, 144-152 (2002).

7 Little, R. D. et al. A mutation in the LDL receptor-related protein 5 gene results in the autosomal dominant high-bone-mass trait. Am J Hum Genet 70, 11-19 (2002).

8 Michael P. Whyte et al. in (LB-1172) American Society for Bone and Mineral Research (ASBMR) 2018 Annual Meeting.

9 Gregson, C. L. et al. Mutations in Known Monogenic High Bone Mass Loci Only Explain a Small Proportion of High Bone Mass Cases. J Bone Miner Res. 31, 640-649 (2015).

10 Gregson, C. L. et al. 'Sink or swim': an evaluation of the clinical characteristics of individuals with high bone mass. Osteo Int. 23, 643-654 (2012).

11 Gregson, C. L. et al. Genome-wide association study of extreme high bone mass: Contribution of common genetic variation to extreme BMD phenotypes and potential novel BMD-associated genes. Bone 114, 62-71 (2018).

12 Kumar, P., Henikoff, S. \& Ng, P. C. Predicting the effects of coding non-synonymous variants on protein function using the SIFT algorithm. Nat Protoc. 4, 1073-1081 (2009).

13 Adzhubei, l. A. et al. A method and server for predicting damaging missense mutations. Nat Meth 7, 248-249 (2010).

14 Schwarz, J. M., Rodelsperger, C., Schuelke, M. \& Seelow, D. MutationTaster evaluates disease-causing potential of sequence alterations. Nat Meth 7, 575-576 (2010).

15 Ferrer-Costa, C. et al. PMUT: a web-based tool for the annotation of pathological mutations on proteins. Bioinformatics 21, 3176-3178 (2005).

16 Zheng, H. F. et al. Whole-genome sequencing identifies EN1 as a determinant of bone density and fracture. Nature 526, 112-11 (2015).

17 Gregson, C. L. et al. The high bone mass phenotype is characterised by a combined cortical and trabecular bone phenotype: Findings from a pQCT case-control study. Bone 52, 380-388 (2013).

18 Morris, J. A. et al. An atlas of genetic influences on osteoporosis in humans and mice. Nature Genetics 51, 258-266 (2019).

19 Staley, J. R. et al. PhenoScanner: a database of human genotype-phenotype associations. Bioinformatics 32, 3207-3209 (2016).

20 Dallas, S. L. \& Bonewald, L. F. Dynamics of the transition from osteoblast to osteocyte. Ann N Y Acad Sci 1192, 437-443 (2010).

21 Youlten S et al. in American Society for Bone and Mineral Research (http://www.asbmr.org/education/2017-abstracts, Denver, Colarado, 2017). 
22 Bergen, D. J. M., Kague, E. \& Hammond, C. L. Zebrafish as an Emerging Model for Osteoporosis: A Primary Testing Platform for Screening New Osteo-Active Compounds. 10, doi:10.3389/fendo.2019.00006 (2019).

23 Tsukamoto, S. et al. Smad9 is a new type of transcriptional regulator in bone morphogenetic protein signaling. Sci Rep 4, 7596, doi:10.1038/srep07596 (2014).

24 Butler, W. T., Mikulski, A., Urist, M. R., Bridges, G. \& Uyeno, S. Noncollagenous proteins of a rat dentin matrix possessing bone morphogenetic activity. Journal of dental research $\mathbf{5 6}$, 228-232 (1977).

25 Lowery, J. W. \& Rosen, V. The BMP Pathway and Its Inhibitors in the Skeleton. Physiol Rev 98, 2431-2452 (2018).

26 Kemp, J. P. et al. Identification of 153 new loci associated with heel bone mineral density and functional involvement of GPC6 in osteoporosis. Nat Genet 49, 1468-1475 (2017).

27 Medina-Gomez, C. et al. Life-Course Genome-wide Association Study Meta-analysis of Total Body BMD and Assessment of Age-Specific Effects. Am J Hum Genet 102, 88-102 (2018).

28 Koay, M. A. et al. Influence of LRP5 Polymorphisms on Normal Variation in BMD. J Bone Miner Res. 19, 1619-1627 (2004).

29 Uitterlinden, A. G. et al. Polymorphisms in the sclerosteosis/van Buchem disease gene (SOST) region are associated with bone-mineral density in elderly whites. Am J Hum Genet 75, 1032-1045 (2004).

30 Boyden, L. M. et al. High bone density due to a mutation in LDL-receptor-related protein 5. New Eng J Med. 346, 1513-1521 (2002).

31 Hamersma, H., Gardner, J. \& Beighton, P. The natural history of sclerosteosis. Clinical Genetics 63, 192-197 (2003).

32 Frost, M. et al. Levels of serotonin, sclerostin, bone turnover markers as well as bone density and microarchitecture in patients with high bone mass phenotype due to a mutation in Lrp5. J Bone Miner Res. 26, 1721-1728 (2011).

33 Gregson, C. L. et al. Elevated Circulating Sclerostin Concentrations in Individuals With High Bone Mass, With and Without LRP5 Mutations. J Clin Endocrinol Metab. 99, 2897-2907 (2014).

34 Bonewald, L. Use it or lose it to age: A review of bone and muscle communication. Bone 120, 212-218 (2018).

35 Shintani, M., Yagi, H., Nakayama, T., Saji, T. \& Matsuoka, R. A new nonsense mutation of SMAD8 associated with pulmonary arterial hypertension. J Med Genet 46, 331-337 (2009).

36 Drake, K. M. et al. Altered MicroRNA processing in heritable pulmonary arterial hypertension: an important role for Smad-8. American journal of respiratory and critical care medicine 184, 1400-1408 (2011).

37 Walcott, B. P. et al. Identification of a rare BMP pathway mutation in a non-syndromic human brain arteriovenous malformation via exome sequencing. Human Genome Variation 5, 18001 (2018).

38 Ngeow, J. et al. Exome Sequencing Reveals Germline SMAD9 Mutation That Reduces Phosphatase and Tensin Homolog Expression and Is Associated With Hamartomatous Polyposis and Gastrointestinal Ganglioneuromas. Gastroenterology 149, 886-889.e885 (2015).

39 Le Goff, C. et al. Mutations in the TGF $\beta$ Binding-Protein-Like Domain 5 of FBN1 Are Responsible for Acromicric and Geleophysic Dysplasias. The American Journal of Human Genetics 89, 7-14 (2011).

40 Loeys, B. L. et al. Mutations in fibrillin-1 cause congenital scleroderma: stiff skin syndrome. Science translational medicine 2, 23ra20, doi:10.1126/scitranslmed.3000488 (2010).

41 Rommel, K. et al. Identification of 29 novel and nine recurrent fibrillin-1 (FBN1) mutations and genotype-phenotype correlations in 76 patients with Marfan syndrome. Hum Mutat 26, 529-539 (2005). 
42 Couser, N. L. et al. The phenotype of multiple congenital anomalies-hypotonia-seizures syndrome 1: report and review. Am J Med Genet A 167a, 2176-2181 (2015).

43 Mclnerney-Leo, A. M. et al. Fryns Syndrome Associated with Recessive Mutations in PIGN in two Separate Families. Hum Mutat 37, 695-702 (2016).

44 Timberlake, A. T. et al. Two locus inheritance of non-syndromic midline craniosynostosis via rare SMAD6 and common BMP2 alleles. eLife 5, doi:10.7554/eLife.20125 (2016).

45 Shore, E. M. et al. A recurrent mutation in the BMP type I receptor ACVR1 causes inherited and sporadic fibrodysplasia ossificans progressiva. Nat Genet 38, 525-527 (2006).

46 Barruet, E. et al. NF-KB/MAPK activation underlies ACVR1-mediated inflammation in human heterotopic ossification. JCl insight 3, e122958, doi:10.1172/jci.insight.122958 (2018).

47 Sheu, T. J. et al. Decreased BMP2 signal in GIT1 knockout mice slows bone healing. 397, 6774 (2014).

48 Hankenson, K. D., Gagne, K. \& Shaughnessy, M. Extracellular signaling molecules to promote fracture healing and bone regeneration. Advanced drug delivery reviews 94, 3-12 (2015).

49 Salazar, V. S., Gamer, L. W. \& Rosen, V. BMP signalling in skeletal development, disease and repair. Nat Rev Endocrinol 12, 203-221 (2016).

50 Duncan, E. L., Cardon, L. R., Sinsheimer, J. S., Wass, J. A. \& Brown, M. A. Site and gender specificity of inheritance of bone mineral density. J Bone Miner Res. 18, 1531-1538 (2003).

51 Gregson, C. L. et al. Analysis of body composition in individuals with high bone mass reveals a marked increase in fat mass in women but not men. J Clin Endocrinol Metab. 98, 818-828 (2013).

52 Ward, K. A., Adams, J. E. \& Hangartner, T. N. Recommendations for thresholds for cortical bone geometry and density measurement by peripheral quantitative computed tomography. Calcif Tissue Int 77, 275-280 (2005).

53 Stratec, Medizintechnik \& GmbH. XCT 2000 Research Manual Software Version 6.20. (2012).

54 The 59th General Assembly Seoul. World Medical Assembly Declaration of Helsinki - Ethical Principles for Medical Research Involving Human Subjects., (Seoul, Korea 2008). Duncan, E. L. et al. Genome-Wide Association Study Using Extreme Truncate Selection Identifies Novel Genes Affecting Bone Mineral Density and Fracture Risk. PLoS Genet 7, e1001372 (2011).

56 Gluer, C. C. et al. Association of five quantitative ultrasound devices and bone densitometry with osteoporotic vertebral fractures in a population-based sample: the OPUS Study. J Bone Miner Res, 19, 782-793 (2004).

57 McCloskey, E. et al. Clodronate reduces vertebral fracture risk in women with postmenopausal or secondary osteoporosis: results of a double-blind, placebo-controlled 3year study. J Bone Miner Res, 19, 728-736 (2004).

58 Simons, L. A. et al. The Dubbo study: an Australian prospective community study of the health of elderly. Australian and New Zealand journal of medicine 20, 783-789 (1990).

59 Mclnerney-Leo, A. M. et al. Short-rib polydactyly and Jeune syndromes are caused by mutations in WDR60. Am J Hum Genet 93, 515-523 (2013).

$60 \mathrm{Li}, \mathrm{H}$. \& Homer, N. A survey of sequence alignment algorithms for next-generation sequencing. Briefings in bioinformatics 11, 473-483 (2010).

$61 \mathrm{Li}, \mathrm{H}$. et al. The Sequence Alignment/Map format and SAMtools. Bioinformatics 25, 20782079 (2009).

62 McKenna, A. et al. The Genome Analysis Toolkit: a MapReduce framework for analyzing next-generation DNA sequencing data. Genome Res 20, 1297-1303 (2010).

63 DePristo, M. A. et al. A framework for variation discovery and genotyping using nextgeneration DNA sequencing data. Nat Genet 43, 491-498 (2011).

64 Wang, K., Li, M. \& Hakonarson, H. ANNOVAR: functional annotation of genetic variants from high-throughput sequencing data. Nucleic acids research 38, e164 (2010). 
65 Abecasis, G. R. et al. A map of human genome variation from population-scale sequencing. Nature 467, 1061-1073 (2010).

66 Albers, C. A. et al. Dindel: accurate indel calls from short-read data. Genome Res 21, 961-973 (2011).

67 de Leeuw, C. A., Mooij, J. M., Heskes, T. \& Posthuma, D. MAGMA: generalized gene-set analysis of GWAS data. PLoS computational biology 11, e1004219 (2015).

68 Pruim, R. J. et al. LocusZoom: regional visualization of genome-wide association scan results. Bioinformatics 26, 2336-2337, doi:10.1093/bioinformatics/btq419 (2010).

69 Hart, T., Komori, H. K., LaMere, S., Podshivalova, K. \& Salomon, D. R. Finding the active genes in deep RNA-seq gene expression studies. BMC Genomics 14, 778 (2013).

70 Zimmermann, L. et al. A Completely Reimplemented MPI Bioinformatics Toolkit with a New HHpred Server at its Core. J Mol Biol 430, 2237-2243 (2018).

71 Webb, B. \& Sali, A. Comparative Protein Structure Modeling Using MODELLER. Current protocols in bioinformatics 54, 5.6.1-5.6.37 (2016).

72 Pettersen, E. F. et al. UCSF Chimera--a visualization system for exploratory research and analysis. Journal of computational chemistry 25, 1605-1612 (2004).

73 Alexander, C. et al. Combinatorial roles for BMPs and Endothelin 1 in patterning the dorsalventral axis of the craniofacial skeleton. Development (Cambridge, England) 138, 5135-5146 (2011).

74 Spoorendonk, K. M. et al. Retinoic acid and Cyp26b1 are critical regulators of osteogenesis in the axial skeleton. 135, 3765-3774 (2008).

75 Westerfield, M. The zebrafish book. A guide for the laboratory use of zebrafish (Danio rerio). Vol. 4th ed. (2000).

76 Hammond, C. L. \& Schulte-Merker, S. Two populations of endochondral osteoblasts with differential sensitivity to Hedgehog signalling. Development (Cambridge, England) 136, 39914000 (2009).

77 Schindelin, J. et al. Fiji: an open-source platform for biological-image analysis. Nature methods 9, 676-682 (2012). 
Table 1: Characteristics of the c.65T>C, p.Leu22Pro SMAD9 HBM pedigree members and two further unrelated HBM individuals with the same SMAD9 mutation

\begin{tabular}{|c|c|c|c|c|c|c|}
\hline & \multicolumn{4}{|c|}{ HBM Pedigree } & \multicolumn{2}{|c|}{$\begin{array}{c}\text { Additional Isolated HBM } \\
\text { cases }\end{array}$} \\
\hline & $\begin{array}{l}\text { UK } \\
\text { proband } \\
\text { III.1 }\end{array}$ & $\begin{array}{l}\text { UK half } \\
\text { sister } \\
\text { III. } 2\end{array}$ & $\begin{array}{l}\text { UK } \\
\text { mother } \\
\text { II. } 2\end{array}$ & $\begin{array}{l}\text { UK grand- } \\
\text { mother } \\
\text { I.2 (unaffected) }\end{array}$ & UK case & $\begin{array}{l}\text { Australian } \\
\text { case }\end{array}$ \\
\hline SMAD9 Mutation & Leu22Pro & Leu22Pro & Leu22Pro & WT & Leu22Pro & Leu22Pro \\
\hline $\begin{array}{l}\text { Age at assessment } \\
\text { Sex } \\
\text { Ethnicity }\end{array}$ & $\begin{array}{l}33 \\
\text { Female } \\
\text { Caucasian }\end{array}$ & $\begin{array}{l}22 \\
\text { Female } \\
\text { Caucasian }\end{array}$ & $\begin{array}{l}55 \\
\text { Female } \\
\text { Caucasian }\end{array}$ & $\begin{array}{l}75 \\
\text { Female } \\
\text { Caucasian }\end{array}$ & $\begin{array}{l}55 \\
\text { Female } \\
\text { Caucasian }\end{array}$ & $\begin{array}{l}57 \\
\text { Female } \\
\text { Caucasian }\end{array}$ \\
\hline \multicolumn{7}{|l|}{ Anthropometry } \\
\hline $\begin{array}{l}\text { Height }(\mathrm{cm}) \\
\text { Weight }(\mathrm{kg}) \\
\text { BMI }\left(\mathrm{kg} / \mathrm{m}^{2}\right)\end{array}$ & $\begin{array}{l}178.0 \\
138.0 \\
43.6 \\
\end{array}$ & $\begin{array}{l}173.3 \\
133.8 \\
43.3 \\
\end{array}$ & $\begin{array}{l}175.0 \\
127.8 \\
41.4 \\
\end{array}$ & $\begin{array}{l}160.6 \\
72.6 \\
28.1 \\
\end{array}$ & $\begin{array}{l}160.0 \\
89.7 \\
35.0 \\
\end{array}$ & $\begin{array}{l}162.2 \\
69.9 \\
26.6 \\
\end{array}$ \\
\hline \multicolumn{7}{|l|}{ DXA Measurements } \\
\hline $\begin{array}{l}\text { Total Hip BMD Z- } \\
\text { score } \\
\text { L1 BMD Z-score } \\
\text { BMC (kg) } \\
\text { Fat mass }(\mathrm{kg}) \\
\text { Lean mass }(\mathrm{kg})\end{array}$ & $\begin{array}{l}3.2 \\
4.5 \\
3.49 \\
73.2 \\
61.3 \\
\end{array}$ & $\begin{array}{l}4.8 \\
2.6 \\
3.77 \\
64.5 \\
65.6 \\
\end{array}$ & \begin{tabular}{l|}
3.3 \\
3.3 \\
3.65 \\
64.8 \\
59.5 \\
\end{tabular} & $\begin{array}{l}0.1 \\
0.8 \\
2.12 \\
25.4 \\
45 \\
\end{array}$ & $\begin{array}{l}4.3 \\
5.0 \\
3.24 \\
34.0 \\
52.4 \\
\end{array}$ & $\begin{array}{l}2.7 \\
+3.4^{*} \\
- \\
- \\
- \\
\end{array}$ \\
\hline \multicolumn{7}{|l|}{ Clinical phenotype } \\
\hline $\begin{array}{l}\text { Adult Fracture } \\
\text { Sinks/ Floats } \\
\text { Bone pain } \\
\text { Visual/auditory } \\
\text { impairment } \\
\text { Dentition } \\
\text { Shoe Size } \\
\text { Broad Frame } \\
\text { Enlarged Mandible } \\
\text { Torus } \\
\text { Nerve } \\
\text { compression } \\
\text { Pes planus } \\
\end{array}$ & $\begin{array}{l}\text { No } \\
\text { Floats } \\
\text { Yes } \\
\text { Myopia } \\
\text { Normal } \\
10 \\
\text { Yes } \\
\text { Yes } \\
\text { Yes } \\
\text { No } \\
\text { No } \\
\end{array}$ & $\begin{array}{l}\text { No } \\
\text { Sinks } \\
\text { Yes } \\
\text { No } \\
\text { Normal } \\
10 \\
\text { Yes } \\
\text { Yes } \\
\text { Yes } \\
\text { No } \\
\text { No } \\
\end{array}$ & $\begin{array}{l}\text { No } \\
\text { Floats } \\
\text { Yes } \\
\text { No } \\
\text { Normal } \\
9 \\
\text { Yes } \\
\text { Yes } \\
\text { No } \\
\text { No } \\
\text { Yes } \\
\end{array}$ & $\begin{array}{l}\text { No } \\
\text { Floats } \\
\text { No } \\
\text { Impaired } \\
\text { hearing } \\
\text { Normal } \\
6 \\
\text { No } \\
\text { No } \\
\text { No } \\
\text { No } \\
\text { No } \\
\end{array}$ & $\begin{array}{l}\text { No } \\
\text { Sinks } \\
\text { No } \\
\text { Astigmatism } \\
\text { Retained } \\
\text { cuspid tooth } \\
5.5 \\
\text { Yes } \\
\text { Yes } \\
\text { No } \\
\text { No } \\
\text { Yes }\end{array}$ & $\begin{array}{l}\text { No } \\
- \\
- \\
- \\
- \\
- \\
- \\
- \\
- \\
- \\
-\end{array}$ \\
\hline \multicolumn{7}{|l|}{ Blood tests } \\
\hline $\begin{array}{l}\text { ALP (IU/L) } \\
\text { Adjusted calcium } \\
\text { (mmol/L) } \\
\text { P1NP (ug/L) } \\
\text { CTX (ug/L) } \\
\text { Osteocalcin (ug/L) } \\
\text { Sclerostin (pmol/L) }\end{array}$ & $\begin{array}{l}99 \\
2.50 \\
58 \\
0.15 \\
12.4 \\
71.0 \\
\end{array}$ & $\begin{array}{l}83 \\
2.46 \\
36 \\
0.18 \\
17.1 \\
- \\
\end{array}$ & $\begin{array}{l}102 \\
2.40 \\
22 \\
0.19 \\
13.5 \\
56.1 \\
\end{array}$ & $\begin{array}{l}202 \\
2.46 \\
95 \\
0.10 \\
14.8 \\
44.4 \\
\end{array}$ & $\begin{array}{l}61 \\
2.33 \\
35 \\
0.16 \\
11.5 \\
50.4 \\
\end{array}$ & $\begin{array}{l}- \\
- \\
- \\
- \\
- \\
-\end{array}$ \\
\hline
\end{tabular}

WT: Wild type. ${ }^{a}$ UK measurements. Reference ranges; ALP 20-120, Adjusted calcium 2.25-2.70, P1NP: premenopausal 30-78 ug/L, postmenopausal 26-110 ug/L, male 20-76 ug/L. Serum CTX 0.1-0.5ug/L, Osteocalcin 6.8-32.2ug/L. Sclerostin $<80 \mathrm{pmol} / \mathrm{L}$. 
Table 2: Distal and mid-shaft tibial pQCT measures in High Bone Mass cases compared with female HBM cases without SMAD9, LRP5, LRP4, SOST mutations, and female family controls with normal BMD

\begin{tabular}{|c|c|c|c|c|}
\hline & & $\begin{array}{c}\text { SMAD9 HBM cases } \\
\text { Leu22Pro } \\
n=4 \\
\text { Mean (SD) }\end{array}$ & $\begin{array}{c}\text { WT Female HBM } \\
\text { cases }^{1} \\
n=76 \\
\text { Mean (SD) }\end{array}$ & $\begin{array}{c}\text { Female family controls } \\
\text { with normal BMD² } \\
n=32 \\
\text { Mean (SD) }\end{array}$ \\
\hline & Age (years) & $41.3(16.5)$ & $60.8(12.3)$ & $54.8(13.5)$ \\
\hline \multirow{3}{*}{$\begin{array}{l}\bar{\pi} \\
\stackrel{n}{0} \\
\stackrel{0}{0} \\
\text { 。 }\end{array}$} & Total Bone area $\left(\mathrm{mm}^{2}\right)$ & $1063(125.7)$ & 1163 (125.9) & $1098(130.1)$ \\
\hline & Trabecular BMD $\left(\mathrm{mg} / \mathrm{cm}^{3}\right)$ & $342.3(13.0)$ & $324.3(22.5)$ & $309.1(23.5)$ \\
\hline & Cortical thickness (mm) & $2.13(0.79)$ & $1.04(0.81)$ & $0.89(0.81)$ \\
\hline \multirow{8}{*}{ 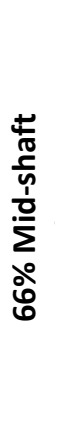 } & Total Bone area $\left(\mathrm{mm}^{2}\right)$ & $608.3(4.7)$ & $603.4(83.1)$ & $574.1(74.7)$ \\
\hline & Cortical BMD (mg/cm³) & $1149.7(10.1)$ & $1126.0(35.6)$ & $1113.8(64.8)$ \\
\hline & Cortical thickness (mm) & $4.96(0.13)$ & $4.36(0.62)$ & $3.85(0.65)$ \\
\hline & Cortical Bone Area $\left(\mathrm{mm}^{2}\right)$ & $356.3(9.1)$ & $316.8(36.3)$ & $277.7(37.6)$ \\
\hline & Cortical/Total Bone area (\%) & $58.6(1.1)$ & $53.2(7.59)$ & $48.9(7.79)$ \\
\hline & $\mathrm{SSI}\left(\mathrm{mm}^{3}\right)$ & $1680(21.1)$ & 1511 (238.5) & $1315.6(231.4)$ \\
\hline & Muscle area $\left(\mathrm{mm}^{2}\right)$ & 8334 (536.5) & $6960(990.2)$ & $6596(1005)$ \\
\hline & Muscle density $\left(\mathrm{mg} / \mathrm{cm}^{3}\right)$ & $38.9(5.1)$ & $40.1(4.0)$ & $40.5(2.9)$ \\
\hline
\end{tabular}

HBM: High Bone Mass, BMD: Bone Mineral Density, SD: Standard Deviation, SSI: Strength Strain Index, WT: Wildtype.

${ }^{1}$ Female subgroup (without SMAD9, LRP5, LRP4, SOST mutations) analysed using data previously published, collected and analysed with the same protocols as SMAD9 HBM cases (17).

${ }^{2}$ Mean (SD) $1^{\text {st }}$ Lumbar Vertebra BMD Z-Score +0.36 (1.1), Total Hip BMD Z-score +0.45 (0.76). 


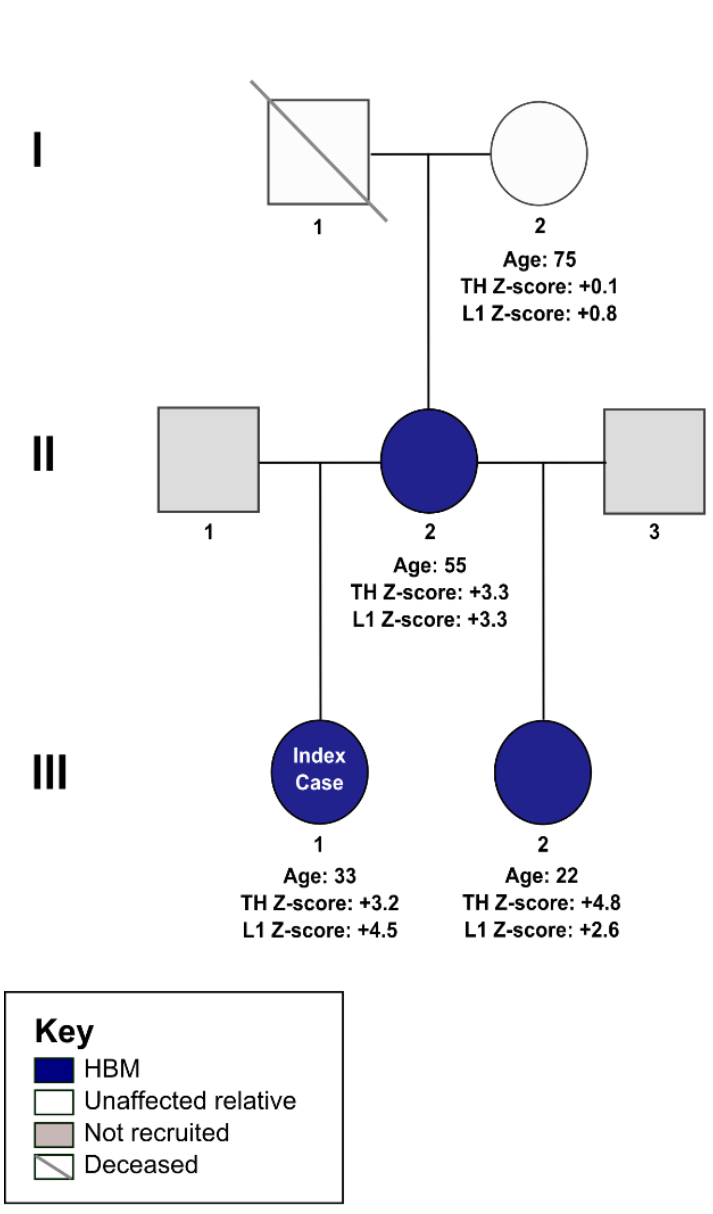

1.2

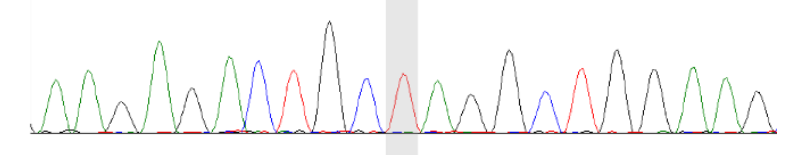

II.2
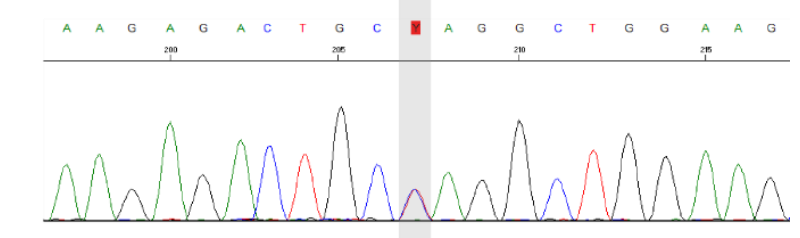

III.1
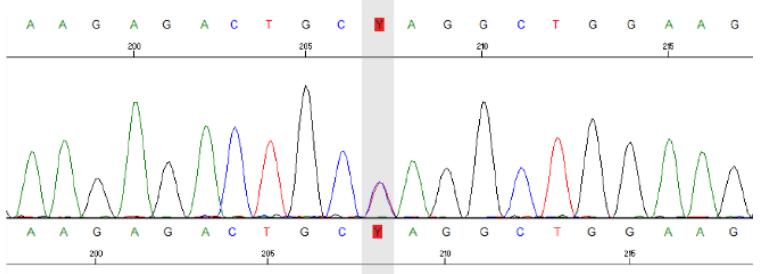

III.2

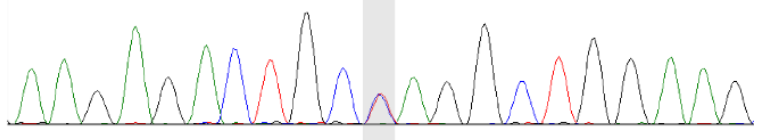

Figure 1: The HBM pedigree and electrophoretogram images of a segregating SMAD9 c.65T>C, p.Leu22Pro variant 

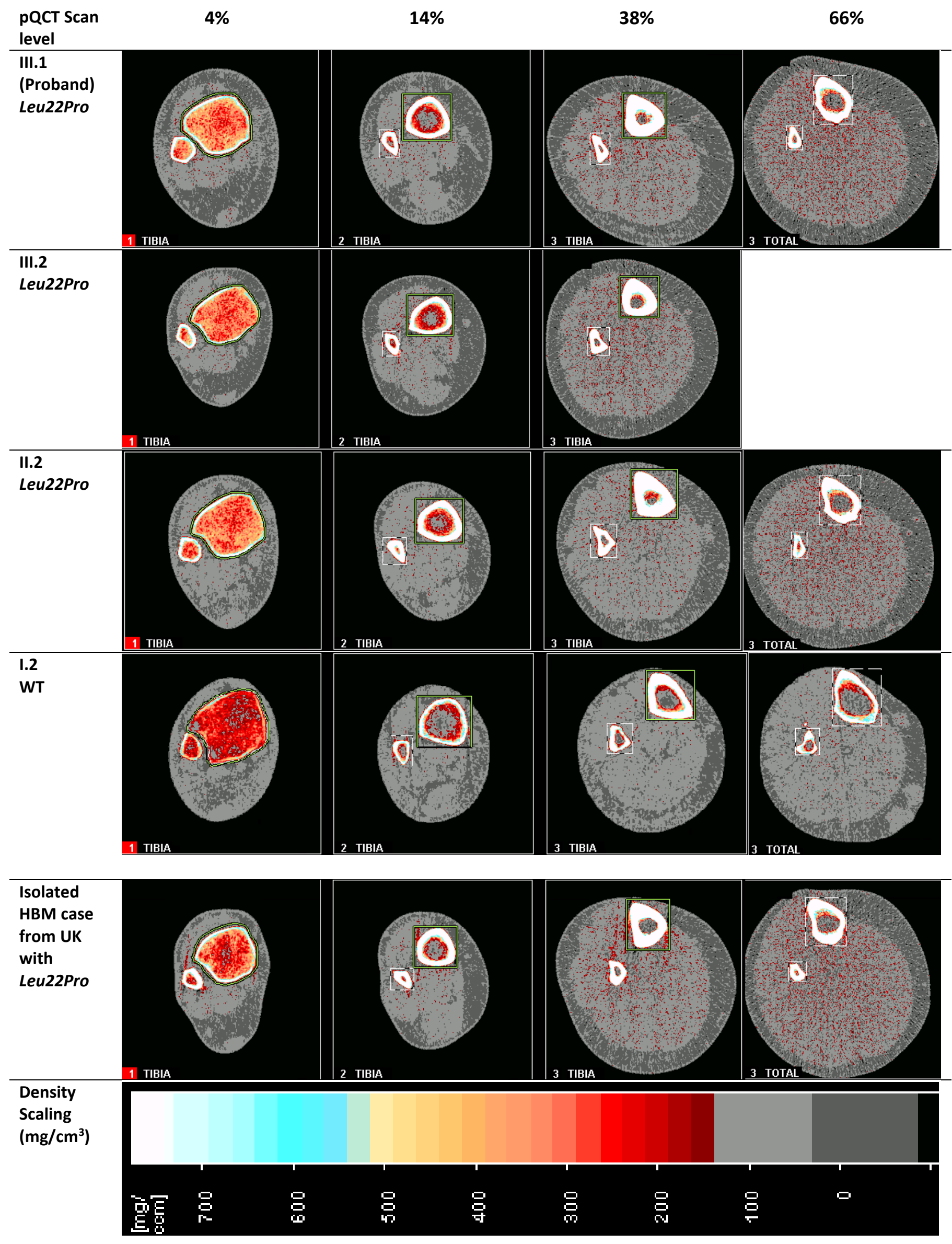
Figure 2 - Tibia pQCT scan results from HBM pedigree and isolated HBM case with Leu22Pro mutation. Images from sequential pQCT scans taken at 4, 14, 38 and $66 \%$ along the tibia from the distal endplate. The $4 \%$ site shows predominantly trabecular bone, whilst cortical bone is thickest at the $38 \%$ site. The $66 \%$ site shows muscle size; of note III. 2 had too large a calf to fit within the pQCT gantry and hence this image was not attained. 
SMAD9

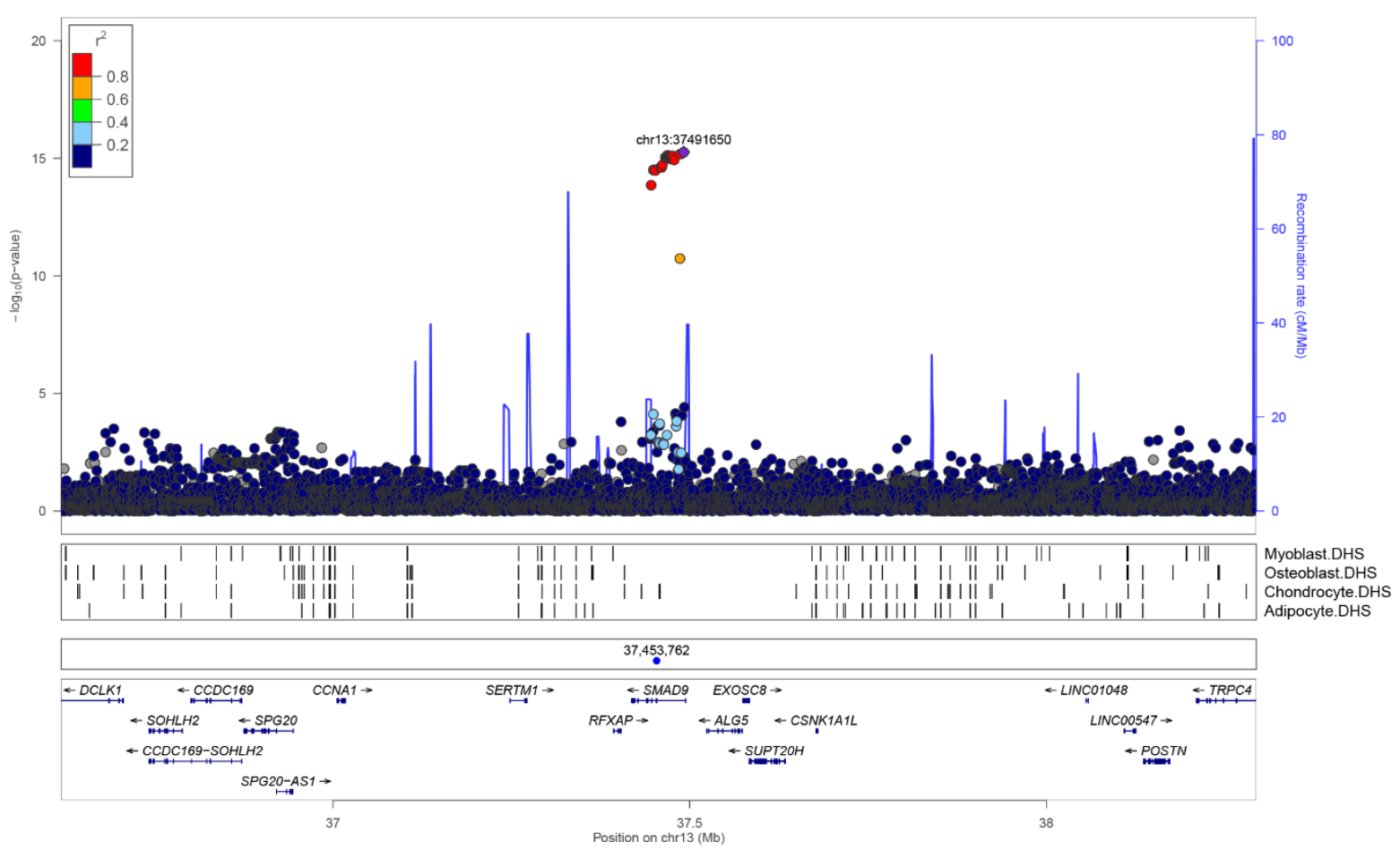

Figure 3-GWAS for eBMD measured in UK Biobank: Regional association plot for the locus containing SMAD9. Top panel: circles show unconditioned GWAS $P$-values and genomic locations of imputed SNPs within +/- 800kb of the 5' and 3' UTR of each gene. Different colours indicate varying degrees of pairwise linkage disequilibrium between the lead eBMD-associated SNP (rs12427846, purple diamond) and all other SNPs. Second panel: Vertical shaded areas correspond to locations of DNase I hypersensitive sites (DHSs) characteristic of: skeletal muscle myoblasts cell line (E120), osteoblast primary cells (E129), mesenchymal stem cell derived chondrocyte cultured cells (E049) and mesenchymal stem cell derived adipocyte cultured cells (E023). Red shading depicts intersections between DHSs and genome-wide significant SNPs. Black shading denotes instances in which any other SNPs intersect DHSs. Third panel: Blue circle shows the position of the putative causal variant c.65T $>C$, p.Leu22Pro. Fifth panel: Horizontal lines represent genes with vertical lines annotating the location of exons. Arrows indicate the direction in which each gene is transcribed. 

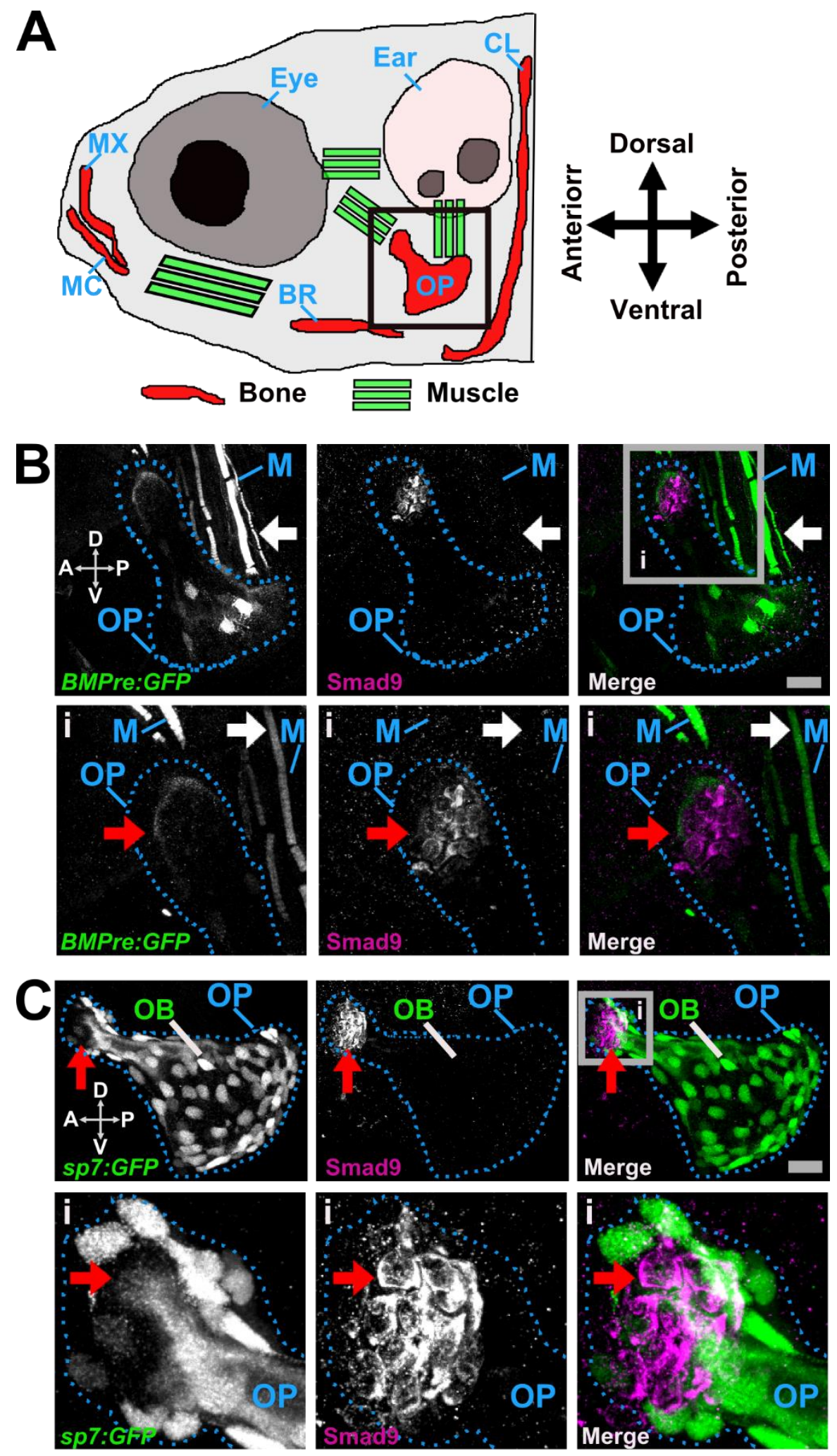

Figure 4-Smad9 protein expression in the larval zebrafish opercle bone

A. Schematic representation of the larval zebrafish head (6 days post fertilisation (dpf), lateral view), showing visible ossified elements (red) and the main muscle groups (green) that are green fluorescent protein (GFP) positive under control of the BMP responsive elements promoter (BMPre) transgenic reporter line (BMPre:GFP). The black box indicates the location of the intramembranous opercle bone as shown in panels $B$ and $C$. 
B. Distinct tissue distribution of Smad9-and BMP-expressing cells (7dpf). Upper left panel: BMPre:GFP positive cells (white) in the levator operculi muscle group (white arrow) and ventral (V) side of the opercle (OP; dotted blue outline); upper middle: distinct group of Smad9 positive cells (white) in the dorsal (D) tip of the opercle; upper right: merged view showing distinct tissue expression of BMPre:GFP positive cells (green) and Smad9 positive cells (purple); lower left: grey box inset (i) showing faint cap of BMPre:GFP positive cells at the dorsal tip of the opercle (red arrow); lower middle: cluster of Smad9 positive cells; lower right: merged view confirming nonoverlapping distribution of BMPre:GFP positive cells and Smad9 positive cells.

C. Distinct tissue distribution of Smad9-and osterix (Sp7)-expressing cells (6dpf). Upper left panel: Sp7:GFP-positive osteoblasts (OB; white) within the opercle; upper middle: Smad9 positive cells (white) in the antero (A)-dorsal tip of the opercle (red arrow); upper right: merged view showing separation of Sp7:GFP positive cells (green) and Smad9 positive cells (purple) (Supplementary Video 1); lower left: the inset ( $i$, grey box) shows few Sp7:GFP-positive osteoblasts within the dorsal tip of the opercle; lower middle: cluster of Smad9 positive cells; lower right: merged view confirming non-overlapping distribution of Sp7:GFP and Smad9 positive cells.

$B$ and $C$ : scale bar is $20 \mu \mathrm{m}$, and all are maximum intensity z-projection confocal images. Abbreviations: $A$, anterior; $B R$, branchiostegal ray; $C L$, cleithrum; $D$, dorsal; $M$, muscle; $M C$, Meckel's cartilage; $\mathrm{MX}$, maxilla; $\mathrm{OB}$, osteoblast; $\mathrm{OP}$, operculum; $\mathrm{P}$, posterior; $\mathrm{V}$, ventral. 


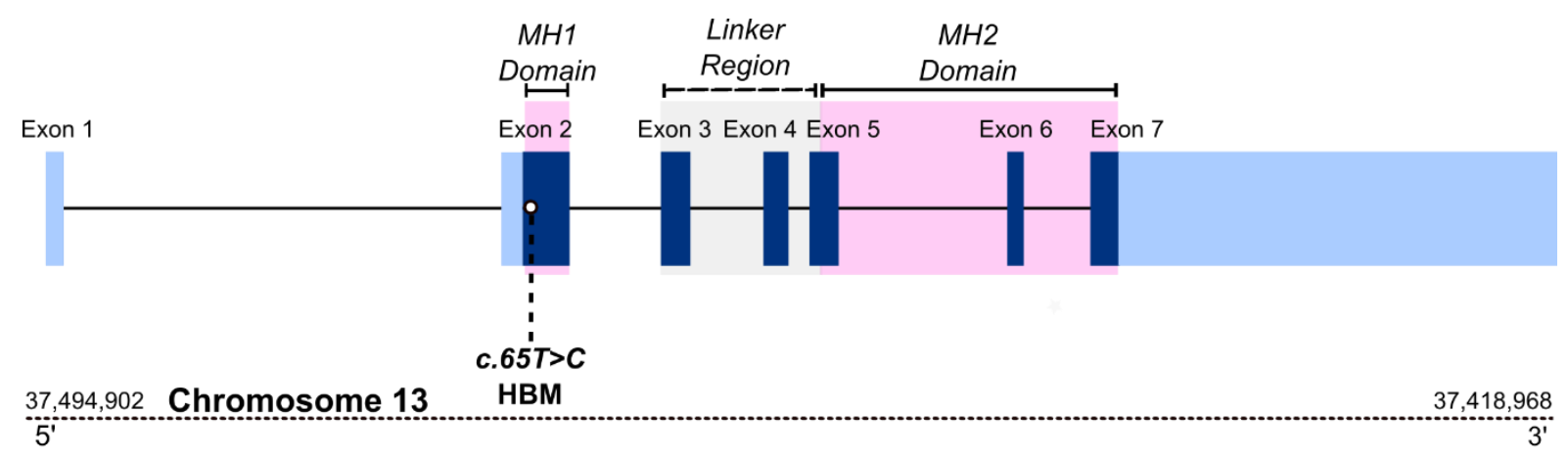

Figure 5 - Figure illustrating position of c.65T>C p.Leu22Pro variant within SMAD9 
Figure 6 (A) Wildtype (WT) SMAD9 MH1 domain (light green ribbon with helix-1 in light blue) binding the DNA helix (dark blue/dark green). L22 is shown in blue space-filling. (B) L22P, shown in red space-filling, is predicted to severely disrupt the structure of the $\mathrm{MH} 1$ domain. Supplementary Video 2: 3-dimensional rotating image

(A) Wild type:

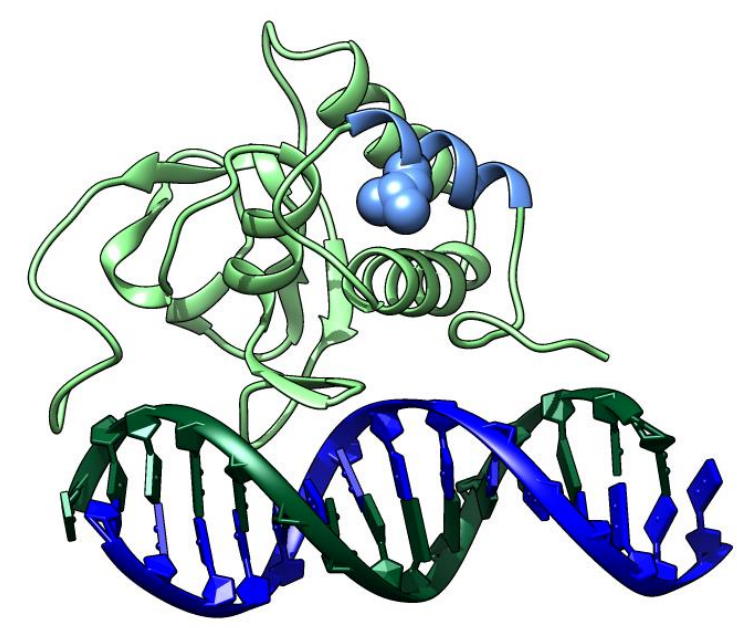

(B) Leu22Pro:

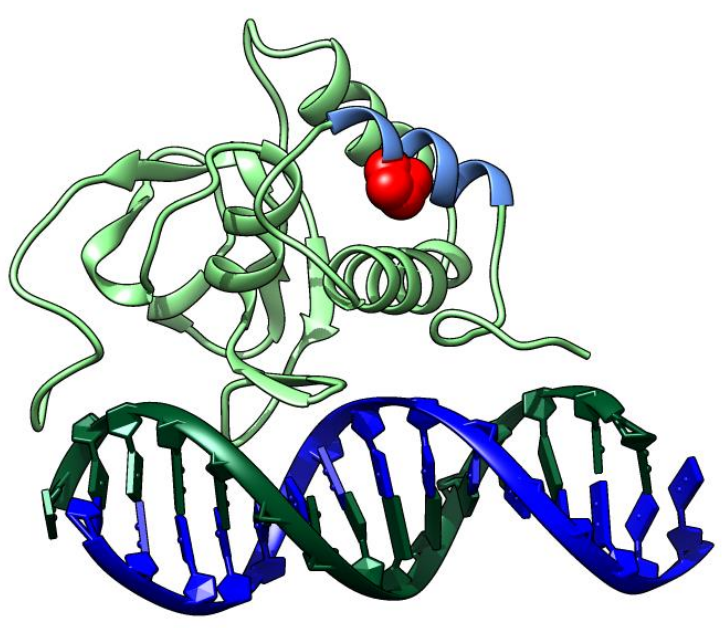

